\title{
Experimental Test of the Siberian Snake Concept
}

\author{
T. Roser \\ Department of Physics, The University of Michigan \\ Ann Arbor, Michigan 48109-1120 USA
}

\begin{abstract}
The Siberian Snake Concept was experimentally tested at the IUCF Cooler Ring. We showed that the spin tune, which is the number of spin rotations per particle revolution, can be manipulated in a predictable way using local spin rotators (Siberian Snakes). Local spin rotators can then be used to avoid depolarization from intrinsic and imperfection depolarizing resonances.
\end{abstract}

I am reporting here on work performed in collaboration with $\mathrm{R}$. Baiod, Ya. S. Derbenev, A. D. Krisch, R. A. Phelps, B. S. van Guilder, and B. Vuaridel ${ }^{*}$ of the University of Michigan; T J P. Ellison, J. E. Goodwin ${ }^{\dagger}$, S. Y. Lee, M. G. Minty, P. V. Pancella ${ }^{*}$, T. Rinckel, M. A. Ross, F. Sperison, E. I. Stephenson, B. von Przewoski of the Indiana University Cyclotron Facility; and E.D. Courant, and L.G. Ratner of Brookhaven National Laboratory.

The Siberian Snake Concept was formulated first by Ya. S. Derbenev and A. M. Kondratenko ${ }^{[1]}$ and states that local spin rotators (Siberian Snakes) can be used to avoid depolarizing resonances in circular accelerators. Before describing our experimental test of this concept I will briefly summarize the current situation of accelerating polarized proton beams.

As is well known, the spin of charged particles precesses around the direction of a magnetic field by an angle, which is $(\mathrm{G} \gamma+1)$ times larger than the angle by which the particle is deflected. $G$ is the anomalous magnetic moment and $\gamma$ is $\mathrm{E} / \mathrm{m}$. As a result of this additional precession the spin will go through $\mathrm{G} \gamma$ full $360^{\circ}$ rotations for every revolution of the particle in a circular accelerator with only vertical magnetic holding fields. This number of full spin precessions per revolution is called 'spin tune', $\nu_{\text {sp }}$. Clearly vertical polarization is stable in such a ring since it coincides with the precession direction. If there are any horizontal magnetic fields present in the accelerator they will rotate the spin away from the vertical direction. Resonant depolarization occurs when these spin rotations add

* Present Address: Département de Physique Nucléaire et Corpusculaire, Université de Genève, CH-1211 Genève 4, Switzerland.

$\dagger$ Present Address: Fermilab, P.O. Box 500, Batavia, IL 60510.

* Present Address: Department of Physics, Western Michigan University, Kalamazoo, MI 49008 . 
coherently in subsequent turns or, in other words, when the precession frequency is equal to the frequency with which the particle experiences spin rotations from horizontal fields.

Magnet misalignments and the vertical focusing fields are two main sources of horizontal fields. They lead to imperfection and intrinsic depolarizing resonances when

$$
\mathrm{n}=\nu_{\mathrm{sp}}=\mathrm{G} \gamma \text { and } \mathrm{n} \pm \nu_{\mathrm{y}}=\nu_{\mathrm{sp}}=\mathrm{G} \gamma
$$

respectively. $\nu_{y}$ is the vertical betatron tune.

Acceleration through these depolarizing resonances without loosing polarization has been achieved at the ZGS, at SATURNE, and at KEK and at the Brookhaven AGS, where polarized protons were accelerated to an energy of 22 $\mathrm{GeV}^{[2]}$. At the AGS 94 small correction dipoles were used to cancel the horizontal fields introduced by magnet misalignments and 10 pulsed quadrupoles were used to shift the betatron tune quickly through the intrinsic resonance condition.

However, these techniques are not practical for the acceleration of polarized protons to much higher energies. Derbenev and Kondratenko ${ }^{[1]}$ first proposed to insert spin rotators into a circular accelerator to avoid depolarization. These so called 'Siberian Snakes' rotate the spin by $180^{\circ}$ around a horizontal direction without changing the particle trajectory. The spin tune $\nu_{\mathrm{sp}}$ with the Siberian Snakes inserted is 0.5 independent of the energy. Clearly both the imperfection and intrinsic resonance conditions are then avoided as long as the fractional part of the vertical betatron tune is not 0.5 .

We conducted a first experimental test of the Siberian Snake Concept at the Indiana University Cyclotron Facility (IUCF) Cooler Ring ${ }^{[3,4]}$. The energy range extends from 30 to $500 \mathrm{MeV}$ and overlaps two depolarizing resonances: an intrinsic resonance at $\mathrm{G} \gamma=\nu_{\mathrm{y}}-3(177 \mathrm{MeV})$ and an imperfection resonance at $\mathrm{G} \gamma=2(108 \mathrm{MeV})$. We used one of the $6 \mathrm{~m}$ long straight sections to install a Siberian Snake. The straight section opposite of the Siberian Snake contained 3 small solenoids which were used to contain the cooling electron beam. These solenoids allowed us to vary the strength of the imperfection resonance. Another straight section was occupied by an internal target set-up with an azimuthally symmetric detector. Using a solid carbon target we were thus able to measure simultaneously both the radial and vertical polarization components. With just one Siberian Snake in the ring the stable polarization direction (or precession direction) lies in the horizontal plane and depends both on energy and location. In Fig. 1 the stable polarization directions are shown for an energy of $108 \mathrm{MeV}$.

Our low energy Siberian Snake consisted of a superconducting solenoid, which rotates the spin by $180^{\circ}$ around the longitudinal direction, a pair of skewed quadrupoles on either side to correct for the spiraling motion introduced by the solenoid and a pair of normal quadrupoles to correct for the introduced focusing. 


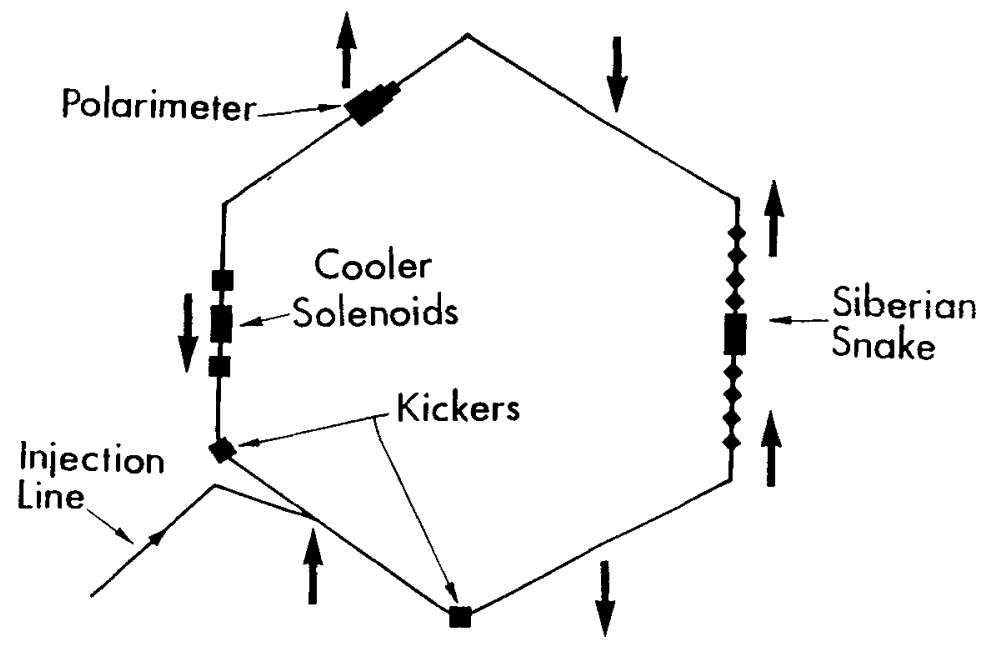

Fig. 1 Schematic diagram of the IUCF Cooler Ring with the Siberian Snake test installed.

Our measurements clearly showed that the depolarizing resonances can be avoided by using the Siberian Snake. Fig. 2 shows polarization measurements at $177 \mathrm{MeV}$ where the nominal betatron tune (5.13) satisfies the intrinsic resonance condition. The measurements without Snake show the expected resonant depolarization, whereas no depolarization was detected with Snake. We studied the $\mathrm{G} \gamma=2$ imperfection resonance by tuning the ring to five energies close to $108 \mathrm{MeV}$, the expected energy of the imperfection resonance. At these energies the stable polarization direction is very sensitive to the strength of the imperfection resonance. This is clearly demonstrated in Fig. 3 which shows both the vertical and radial polarization components measured as a function of the imperfection field generated by the small solenoids in the Cooler straight section. Measurements with Snake on showed no depolarization at any of these five energies and at any imperfection field.

On the right side in Fig. 3 the ratio $\mathrm{P}_{\text {radial }} / \mathrm{P}_{\text {vertical }}$ is plotted. For energies close to the imperfection resonance $P_{\text {radial }} / P_{\text {vertical }}$ is expected to lie on a straight line and the inverse slope is a measure of $\nu_{\mathrm{sp}}-2$. The data presented in Fig. 3 is consistent with

$$
\nu_{\mathrm{sp}}=\mathrm{G} \gamma+0.0036 \pm 0.0003
$$

which is slightly, but significantly different from $\nu_{\mathrm{sp}}=\mathrm{G} \gamma$ expected for a pure vertical holding field. We were able to trace this difference to the quite complicated magnet structure in the Cooler straight section, which effectively added a 


\section{The Siberian Snake Concept}

small spin rotation around the vertical direction ${ }^{[5]}$. Such a magnet configuration had been named a partial type-III Siberian Snake ${ }^{[6]}$.

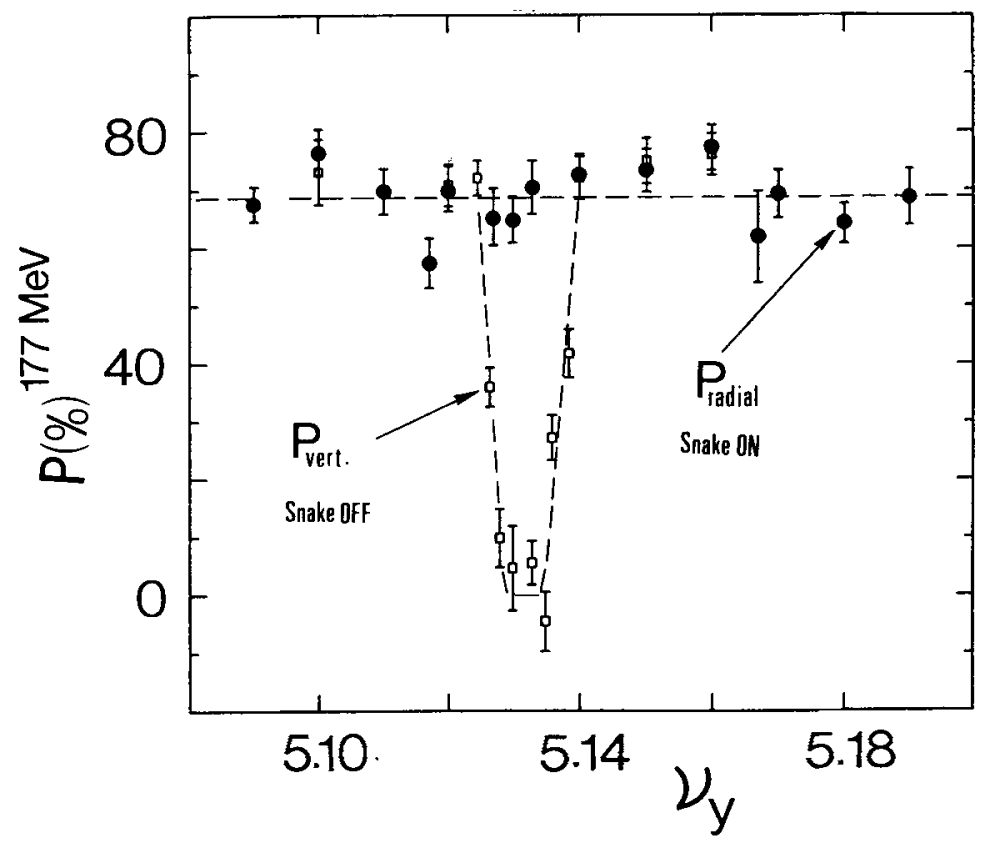

Fig. 2 Polarization measurements with and without Snake at $177 \mathrm{MeV}$ plotted against the vertical betatron tune $\nu_{\mathrm{y}}$.

We were also able to directly measure the spin tune with the Siberian Snake turned on by depolarizing the beam with a RF magnetic field. The spin tune is equal to the ratio of the resonance $R F$ frequency and the revolution frequency. We measured a spin tune of 0.5086 which is in good agreement with the expected value of 0.5 within the calibration uncertainty of the Snake solenoid magnetic field.

For a more quantitative study of the Siberian Snake Concept we studied the general case of a spin rotator that rotates the spin direction by an angle $\delta$, which may lie anywhere between $0^{\circ}$ and $180^{\circ}$. The spin tune for such a 'Partial Siberian Snake' is given by the formula

$$
\cos \left(\pi \nu_{\mathrm{sp}}\right)=\cos (\pi \mathrm{G} \gamma) \cos \left(\frac{\delta}{2}\right)
$$

This equation shows that even for a small spin rotation the spin tune $\nu_{\text {sp }}$ will never be an integer and thus at least imperfection resonances can be avoided. 

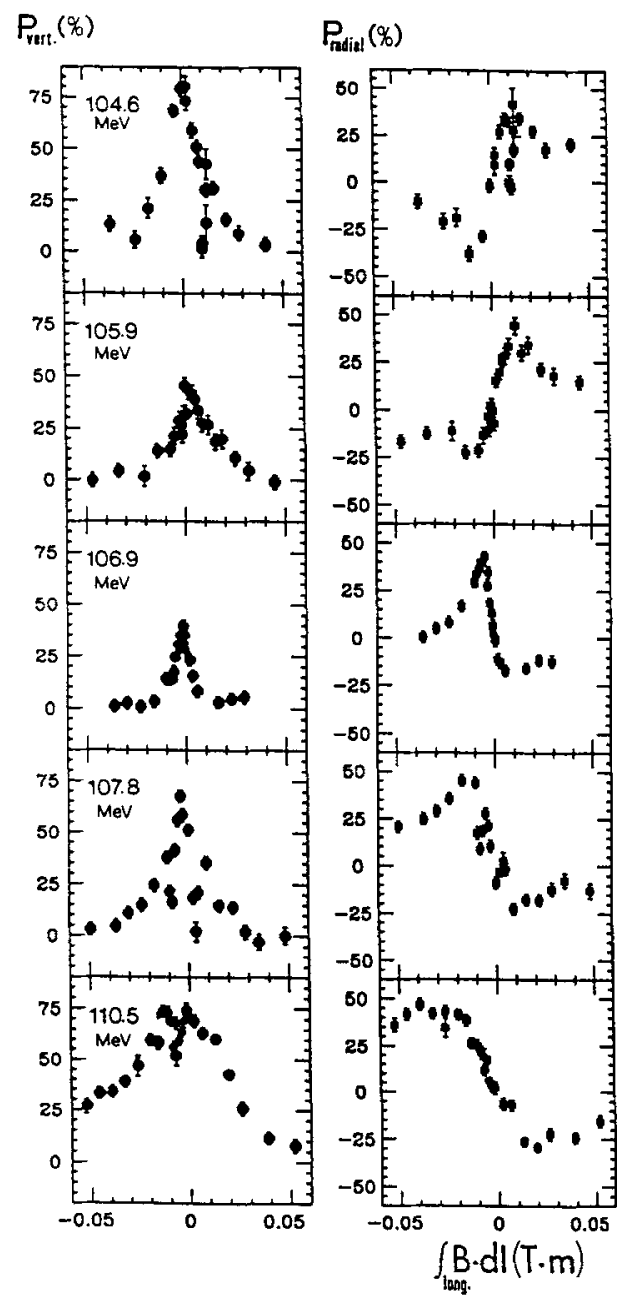

$P_{\text {nitial }} / P_{\text {vitr. }}$

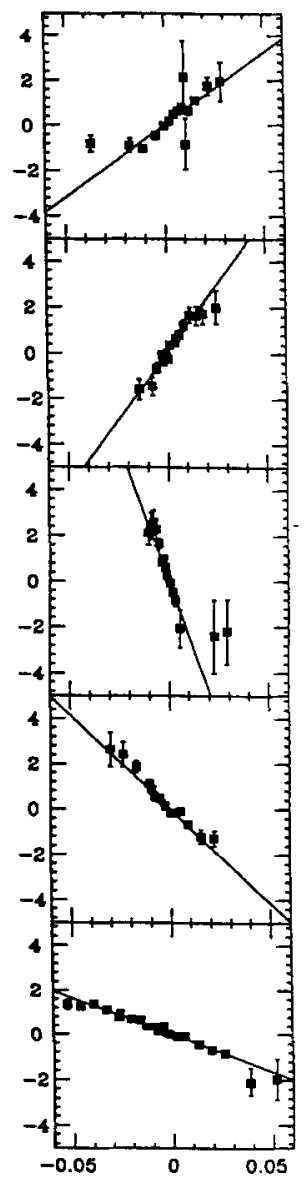

Fig. 3 Measured vertical (left) and radial (center) polarization at 104.6, 105.9, $106.9,107.8$, and $110.5 \mathrm{MeV}$ are plotted against the net longitudinal magnetic field integral in the Cooler section solenoids. The narrow dips in the radial and vertical polarizations are probably due to synchrotron depolarizing resonances ${ }^{[4]}$

To test Eq. (2) we used a $25 \%$ Siberian Snake to observe the $7-\nu_{\mathrm{y}}$ intrinsic resonance. Without a Snake this resonance occurs at about $40 \mathrm{MeV}$. With a $25 \%$ Siberian Snake, however, the resonance $\nu_{\mathrm{sp}}=7-\nu_{\mathrm{y}}$ can be observed at $104 \mathrm{MeV}$, since the partial Snake lowers the spin tune at this energy. Fig. 4 shows our measurements under these conditions with the depolarizing resonance at exactly the location predicted by Eq. (2). 


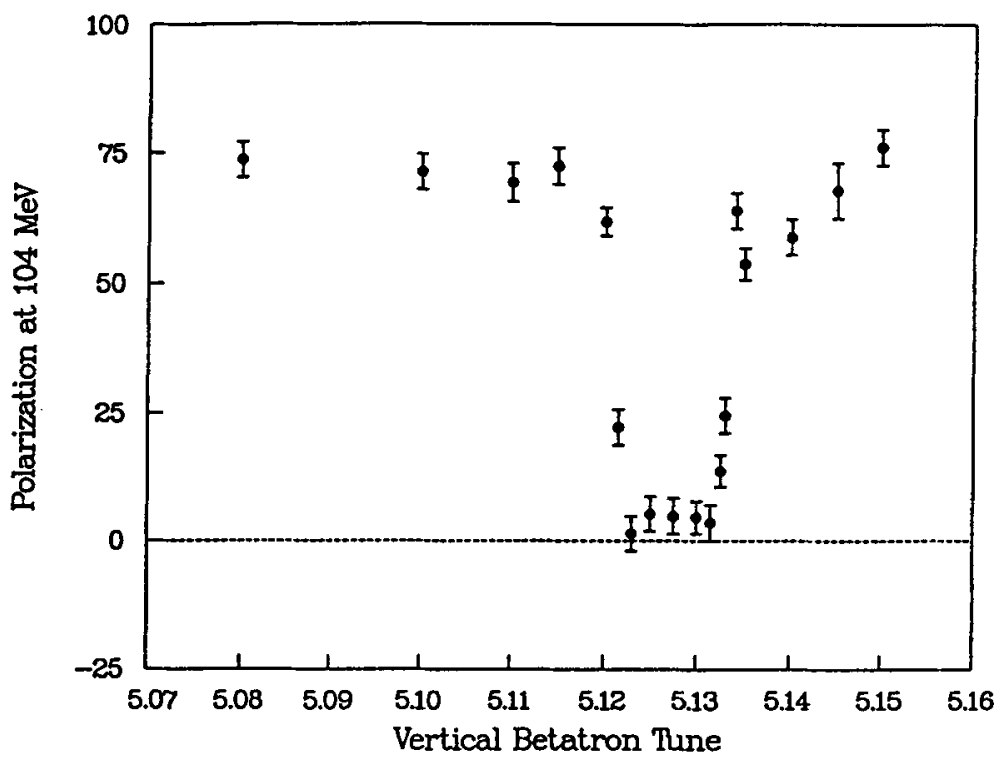

Fig. 4 Polarization measurements with a $25 \%$ Siberian Snake $\left(\delta=45^{\circ}\right)$ at 104 $\mathrm{MeV}$ plotted against the vertical betatron tune, $\nu_{\boldsymbol{y}}$.

From our measurements so far at the IUCF Cooler Ring we can conclude that the spin tune in a circular accelerator can be manipulated by local spin rotators and follows $\mathrm{Eq}(2)$ for a single spin rotator. This ability to control the spin tune can then be used to avoid depolarizing resonances and should allow to accelerate polarized beams to very high energies.

This research was supported by grants from the U.S. Department of Energy and the U.S. National Science Foundation.

\section{REFERENCES}

1. Ya. S. Derbenev and A. M. Kondratenko, Part. Accel. 8, 115 (1978).

2. F. Z. Khiari et al., Phys. Rev. D39, 45 (1989).

3. A. D. Krisch et al., Phys. Rev. Lett. 63, 1137 (1989).

4. J. E. Goodwin et al., Phys. Rev. Lett. 64, 2779 (1990).

5. R. E. Pollock, Nucl. Inst. and Meth. A300, 210 (1991).

6. M. G. Minty et al., Submitted to Phys. Rev. Lett.

7. T. Roser in Proceedings of the $8^{\text {th }}$ International Symposium on High Energy Spin Physics, Minneapolis, 1988, ed. K. Heller, AIP Conf. Proc. No. 187 (New York, 1988) p. 1442. 